\title{
Cultural and Serological Studies on Haemophilus vaginalis
}

\author{
By DONNA L. REDMOND AND E. KOTCHER* \\ Department of Microbiology, School of Medicine, University of Louisville, \\ Louisville 2, Kentucky, U.S.A.
}

(Received 22 March 1963)

\begin{abstract}
SUMMARY
When several strains of Haemophilus vaginalis were grown on Casman rabbit blood agar, individual morphological and cultural differences were noted between the Amies strains which formed pleomorphic and filamentous organisms and large, umbonate colonies, and the Dukes, Edmunds, King and U/L strains which were microscopically coccobacillary to bacillary, non-filamentous, and formed minute convex smooth colonies. Dukes, Edmunds, King and U/L strains required whole blood for maintenance while a whole blood derivative, e.g. peptic digest or Difco chocolate-yeastolate agar, was sufficient for the maintenance of the Amies strains. Serological studies by tube agglutination, direct, indirect and inhibition immunofluorescent methods showed that Dukes, Edmunds, King and $U / L$ strains reacted in a homologous manner with $H$. vaginalis antisera nos. 317, 394 and 4984. Amies strains did not react with these antisera. However, Amies strains cross-reacted with $H$. aegyptius antiserum 180 A, while the Dukes, Edmunds, King and U/L strains did not react with this antiserum.
\end{abstract}

\section{INTRODUCTION}

Several investigators (Leopold, 1953; Gardner \& Dukes, 1954, 1955; Gardner, Dampeer \& Dukes, 1957; Amies \& Jones, 1957; Brewer, Halpern \& Thomas, 1957; Edmunds, $1960 a$; Lapage, 1961 ; and others) have reported finding a small Gramnegative bacillus in the human female and male genito-urinary tract. These investigators used various media for the isolation and cultivation of this organism, named Haemophilus vaginalis by Gardner \& Dukes (1955). Although most of these investigators used the criteria for identification established by Gardner \& Dukes, several reports were at variance with the cultural behaviour and staining characteristics. Amies \& Jones (1957) noted smooth-rough (S-R) colony forms of their strains, and their rough colonies contained more filamentous forms of the organism than did the smooth. No other investigators have reported S-R dissociation of $\boldsymbol{H}$. vaginalis, nor have they reported filamentous forms. Variability in Gram reaction was observed by Brewer et al. (1957),Lutz \& Burger (1957) and Edmunds (1960a). Dukes \& Gardner (1961) reported Gram variability in 72 and $90 \mathrm{hr}$. cultures and associated this with the formation of volutin granules. Edmunds (1960 $a$ ) related Gram variability to volutin granules which he believed to be formed by the fermentation of certain sugars, in particular glucose. Later he found $(1960 b)$ that haemolytic activity

\footnotetext{
* Present address of the authors: International Center for Medical Research and Training, Louisiana State University; School of Medicine, Apartado 5140, San Jose, Costa Rica.
} 
varied with the animal source of red blood cells. On his meat digest agar he obtained optimal haemolysis with human red blood cells whilst lysis of rabbit red blood cells was variable. Dukes \& Gardner (1961) observed a green to brown discoloration type of haemolysis on Casman blood agar medium to be produced by most of the strains investigated. In view of these differences it was decided to make a comparative cultural study of representative strains and to determine whether serological studies might bear out the cultural differences. Strains of $\boldsymbol{H}$. aegyptius and $\boldsymbol{H}$. influenzae were also included in the study.

\section{METHODS}

\section{Organisms used}

Haemophilus vaginalis: strains 317, 394, 513 and 594 from Dr C. D. Dukes; strains н 36, н 76, н 2030 from Dr C. R. Amies; strain т 145 from Dr P. N. Edmunds; strain 4984 (King strain) from Miss E. O. King; strains 842 and 867 (U/L strains) from University of Louisville.

Haemophilus aegyptius: strains $180 \mathrm{~A}$ and $181 \mathrm{~A}$ from Dr M. Pittman.

Haemophilus influenzae: ATCC strains 8412, 9006, 9007, 9008 and 9334 from the American Type Culture Collection.

Media. Casman (1947) broth and agar (Difco) $+5 \%$ fresh defibrinated rabbit blood. Chocolate-yeastolate (Difco) agar (5\% defibrinated rabbit blood and $1 \%$ yeastolate added to heart infusion agar base). Fildes peptic digest agar (1920) with defibrinated rabbit blood and Casman agar or heart infusion agar.

Incubation was at $37^{\circ}$ in a candle jar.

Staining. Smears of Haemophilus vaginalis cultured for up to 5 days in Casman rabbit blood agar were prepared, air dried and heat-fixed. The smears were stained by the potassium mercuric iodide modification of the Gram method, by Giemsa and by basic fuchsin dilute solution. Strains of a vaginal diphtheroid and of Staphylococcus aureus were used as Gram-positive controls.

Antiserum preparation. Antisera for Haemophilus vaginalis strains 317, 394 and 4984, and for $H$. aegyptius strain $180 \mathrm{~A}$ were prepared by inoculating live organisms (3-4 $\times 10^{6}$ bacteria/ml. sterile saline) intravenously, and formalin-killed organisms mixed with Freund adjuvant (Difco) subcutaneously, into the heel pad of normal, healthy New Zealand male rabbits (4-6 lb.) at various times during 24 days. Antisera were obtained one week following the last inoculation by sterile cardiac puncture, and stored at $-60^{\circ}$ for subsequent use. Antisera for $H$. vaginalis strains 317 and 4984 and for $H$. aegyptius strain $180 \mathrm{~A}$ were used to prepare fluoresceinconjugated globulins by the method of Cherry, Goldman, Carski \& Moody (1960). Human liver powder and bovine red bone marrow were used for the adsorption of the globulin conjugates to decrease non-specific fluorescence. Immunofluorescent reactions were carried out by direct, indirect and inhibition methods on air-dried heat-fixed bacterial smears. The fluorescence equipment used in these studies had an HB-200 mercury vapour bulb as a light source, with two Corning 5840 exciter filters in one case, and a BG12 exciter filter in the other.

Agglutination tests. Tube agglutination tests (Evans, 1957) were done with live bacteria of various species and strains derived from $18 \mathrm{hr}$. cultures. The diluent was Hanks balanced salt solution containing $0.1 \%$ bovine serum albumin. The tubes 
were incubated in a $37^{\circ}$ water bath for $3 \mathrm{hr}$. and examined macroscopically and microscopically for agglutination. The tubes were then held at $4^{\circ}$ for $18 \mathrm{hr}$., and again examined for agglutination.

\section{RESULTS}

\section{Morphology and staining reactions}

Smears made from $18 \mathrm{hr}$. Casman rabbit blood agar cultures showed a bacillus ranging from coccobacillary to bacillary forms for the Dukes, King, Edmunds and U/L strains of Haemophilus vaginalis; the three Amies strains when grown on Casman medium showed extreme pleomorphism, with many filamentous forms. The

Table 1. Growth of various strains of Haemophilus vaginalis

\begin{tabular}{lccccc}
\multicolumn{1}{c}{ Strain } & $\begin{array}{c}\text { Casman } \\
\text { rabbit } \\
\text { blood } \\
\text { agar }\end{array}$ & $\begin{array}{c}\text { Casman } \\
\text { rabbit } \\
\text { blood } \\
\text { broth }\end{array}$ & $\begin{array}{c}\text { Fildes } \\
\text { peptic } \\
\text { digest } \\
\text { agar }\end{array}$ & $\begin{array}{c}\text { Chocolate } \\
\text { yeastolate } \\
\text { agar }\end{array}$ \\
Dukes 513 & - & + & + & - & \pm \\
Dukes 594 & - & + & + & - & \pm \\
Eukes 394 & - & + & + & - & \pm \\
King 4984 & - & + & + & - & \pm \\
U/L 867 & - & + & + & - & \pm \\
U/L 842 & - & + & + & + & \pm \\
Amies H76 & - & + & + & + & + \\
Amies H 36 & - & + & + & + & + \\
Amies H 2030 & - & + & + & + & + \\
Pittman 180 A* & - & & + & + & +
\end{tabular}
+ , Growth; \pm , sparse growth (could not be subcultured); - , no growth. * a strain of $H$. aegyptius

Table 2. Tube agglutination reaction

\begin{tabular}{|c|c|c|c|c|c|c|c|c|}
\hline \multirow[b]{3}{*}{ Antigen } & \multicolumn{8}{|c|}{ Reciprocal of agglutination titre with antisera } \\
\hline & \multicolumn{2}{|c|}{$\begin{array}{c}\text { H. vaginalis } \\
\mathbf{3 1 7}\end{array}$} & \multicolumn{2}{|c|}{$\begin{array}{c}H . \text { vaginalis } \\
\mathbf{4 9 8 4}\end{array}$} & \multicolumn{2}{|c|}{$\begin{array}{c}\text { H. vaginalis } \\
\mathbf{3 9 4}\end{array}$} & \multicolumn{2}{|c|}{$\begin{array}{c}H, \text { aegyptius } \\
180 \mathrm{~A}\end{array}$} \\
\hline & $3 \mathrm{hr}$. & $18 \mathrm{hr}$. & $3 \mathbf{h r}$. & $18 \mathrm{hr}$. & 3 hr. & $18 \mathrm{hr}$. & 3 hr. & $18 \mathrm{hr}$. \\
\hline 317 (Dukes) & 5120 & $\mathbf{5 1 2 0}$ & 1280 & 1280 & 640 & 640 & 10 & 10 \\
\hline 513 (Dukes) & 640 & 640 & 320 & 640 & 320 & 320 & 0 & $\mathbf{0}$ \\
\hline 394 (Dukes) & 640 & 640 & 320 & 320 & 2560 & 2560 & 10 & 10 \\
\hline 594 (Dukes) & 320 & 320 & 640 & 640 & 640 & 640 & 0 & $\mathbf{0}$ \\
\hline 4984 (King) & 640 & 640 & 5120 & 5120 & 320 & 320 & $\mathbf{0}$ & $\mathbf{0}$ \\
\hline T 145 (Edmunds) & 320 & 320 & 640 & 1280 & 640 & 1280 & 0 & $\mathbf{0}$ \\
\hline $842(\mathrm{U} / \mathrm{L})$ & 640 & 640 & 320 & 320 & 320 & 320 & $\mathbf{0}$ & $\mathbf{0}$ \\
\hline $867(U / L)$ & 160 & 320 & 1280 & 1280 & 320 & 640 & 0 & $\mathbf{0}$ \\
\hline н 2030 (Amies) & 10 & 10 & 10 & 10 & 10 & 10 & 40 & 80 \\
\hline H7 6 (Amies) & 20 & 10 & 10 & 0 & 0 & 0 & 40 & 80 \\
\hline 180 A (Pittman) & 0 & 10 & 10 & 10 & 0 & $\mathbf{0}$ & 2560 & 2560 \\
\hline 181 A (Pittman) & 0 & $\mathbf{0}$ & 0 & $\mathbf{0}$ & $\mathbf{0}$ & $\mathbf{0}$ & 1280 & 1280 \\
\hline$e 9008(\mathrm{ATCC})$ & 0 & $\mathbf{0}$ & 0 & 0 & 0 & $\mathbf{0}$ & $\mathbf{0}$ & $\mathbf{0}$ \\
\hline 8412 (ATCC) & $\mathbf{0}$ & $\mathbf{0}$ & $\mathbf{0}$ & $\mathbf{0}$ & $\mathbf{0}$ & $\mathbf{0}$ & $\mathbf{0}$ & $\mathbf{0}$ \\
\hline 9006 (ATCC) & 0 & $\mathbf{0}$ & 0 & $\mathbf{0}$ & $\mathbf{0}$ & $\mathbf{0}$ & $\mathbf{0}$ & $\mathbf{0}$ \\
\hline 334 (ATCC) & $\mathbf{0}$ & $\mathbf{0}$ & 0 & 0 & $\mathbf{0}$ & 0 & 0 & $\mathbf{0}$ \\
\hline$e 9007$ (AтCC) & 0 & 0 & 0 & 0 & 0 & $\mathbf{0}$ & 0 & $\mathbf{0}$ \\
\hline
\end{tabular}

H. vaginalis 317 (Dukes)

H. vaginalis 513 (Dukes)

$H$. vaginalis 394 (Dukes)

$H$, vaginalis 594 (Dukes)

$H$. vaginalis 4984, (King)

$H$. vaginalis $\mathrm{T} 145$ (Edmunds)

$H$. vaginalis $842(\mathrm{U} / \mathrm{L})$

$H$. vaginalis $867(\mathrm{U} / \mathrm{L})$

H. vaginalis н 2030 (Amies)

$H$. vaginalis $\mathrm{H} 76$ (Amies)

H. aegyptius $180 \mathrm{~A}$ (Pittman)

H. aegyptius 181 A (Pittman)

H. influenzae 9008 (ATCC)

H. influenzae 8412 (ATCC)

H. influenzae 9006 (ATCC)

H. influenzae 9334 (ATCC)

H. influenzae 9007 (ATCC) 


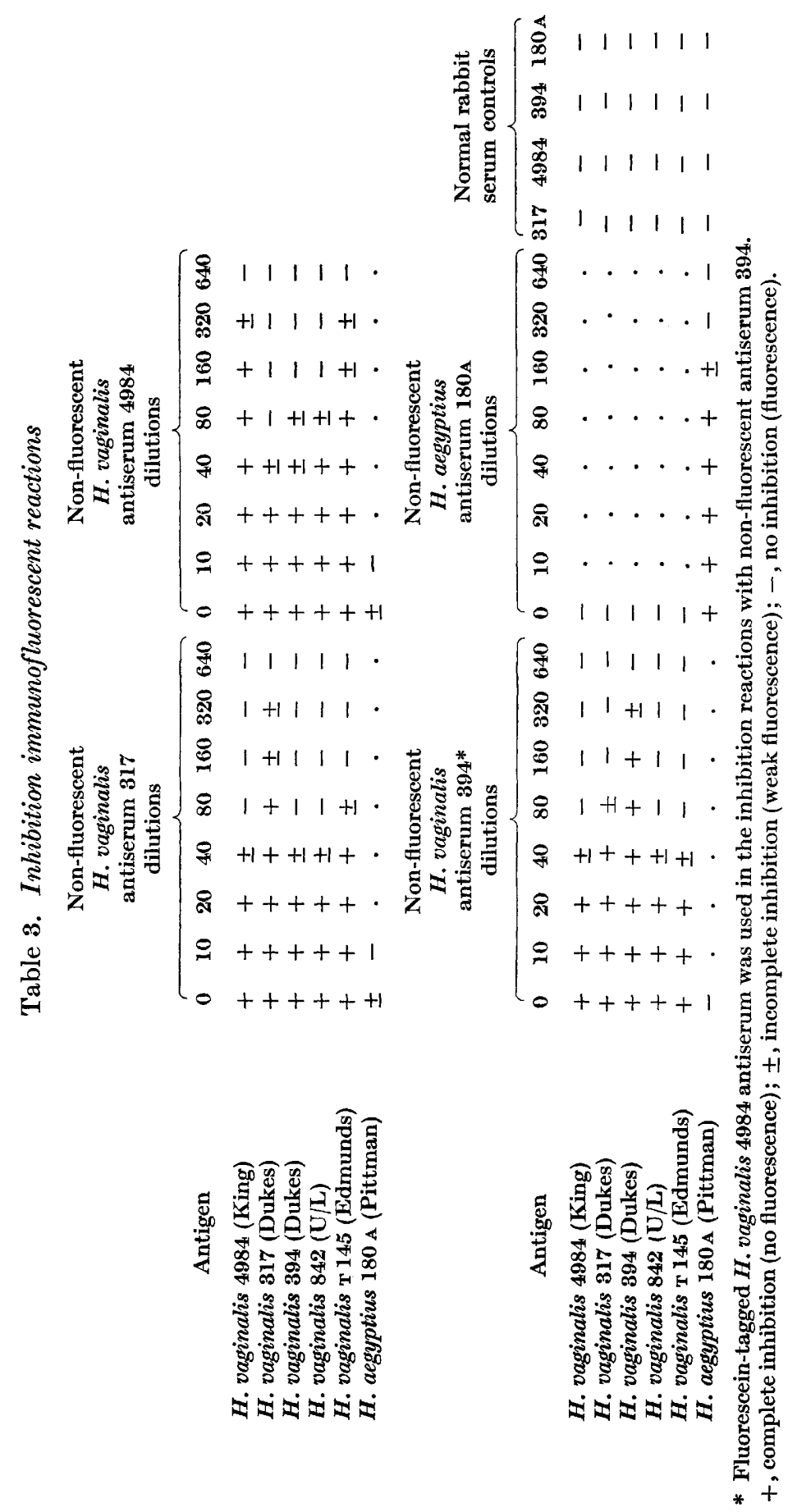


size of basic fuchsin-stained organisms ranged from 0.4-0.6 $\mu \times 1 \cdot 0-2 \cdot 0 \mu$ for the Dukes, King, Edmunds and U/L strains, whereas the Amies strains tended to be slightly longer, $2 \cdot 0-3 \cdot 5 \mu$.

Organisms from such cultures were Gram-negative. Gram variability was noted occasionally with some strains (Edmunds, King) in smears made from 48 and $72 \mathrm{hr}$. In Casman rabbit blood broth cultures, such Gram variability was not observed with the Amies (н 2030), Dukes or U/L (842) strains. Bipolar staining was observed in some Giemsa stained organisms from Casman rabbit blood agar cultures of Dukes, Edmunds, King and U/L strains. Two of the Amies strains (н 36, н 76) did not show bipolar staining, while strain $\mathrm{H} 2030$ showed a few bipolar organisms. The Amies strains showed filamentous forms on Casman rabbit blood agar.

Table 4. Direct immunofluorescent reaction

\begin{tabular}{|c|c|c|c|}
\hline \multirow[b]{2}{*}{ Organism and source } & \multicolumn{3}{|c|}{ Fluorescent antiserum } \\
\hline & $\begin{array}{c}\text { H. vaginalis } \\
\mathbf{3 1 7}\end{array}$ & $\begin{array}{c}H, \text { vaginalis } \\
4984\end{array}$ & $\begin{array}{c}\text { H. aegyptius } \\
180 \mathrm{~A}\end{array}$ \\
\hline H. vaginalis 317 (Dukes) & + & + & - \\
\hline H. vaginalis 394 (Dukes) & + & + & - \\
\hline H. vaginalis 4984 (King) & + & + & - \\
\hline H. vaginalis $\mathrm{T} 104$ (Edmunds) & + & + & - \\
\hline H. vaginalis $\mathrm{T} 145$ (Edmunds) & + & + & - \\
\hline H. vaginalis $680(\mathrm{U} / \mathrm{L})$ & + & + & - \\
\hline H. vaginalis $842(\mathrm{U} / \mathrm{L})$ & + & + & - \\
\hline H. vaginalis 867 (U/L) & + & + & - \\
\hline H. vaginalis н 36 (Amies) & - & - & + \\
\hline H. vaginalis $\mathrm{x} 76$ (Amies) & - & - & \pm \\
\hline H. vaginalis н 2030 (Amies) & - & - & $\bar{t}$ \\
\hline H. aegyptius $180 \mathrm{~A}$ (Pittman) & - & - & + \\
\hline H. aegyptius 181 A (Pittman) & - & - & + \\
\hline H. influenzae 9006 (ATCC) & - & - & + \\
\hline H. influenzae 9007 (ATCC) & - & - & + \\
\hline H. influenzae 9008 (ATCC) & - & - & + \\
\hline H. infuenzae 9334 (ATCC) & - & - & + \\
\hline H. influenzae 8142 (ATCC) & - & - & + \\
\hline
\end{tabular}

+ , fluorescence; \pm , weak fluorescence; - , no fluorescence.

Colonial morphology. The colonial morphology of the various Haemophilus vaginalis strains on Casman rabbit blood agar were studied after $24 \mathrm{hr}$. and $48 \mathrm{hr}$. incubation in a candle jar. The Dukes, Edmunds, King and U/L strains formed colonies like those described by Dukes \& Gardner (1961). The Amies strains, however, were umbonate, entire, translucent and slightly granular, varying in size from $0.37-0.97 \mathrm{~mm}$. in diameter. They were similar in this respect to $H$. aegyptius strain $180 \mathrm{~A}$.

Haemolytic activity on rabbit red cells in Casman medium showed a wide range of variability from no reaction to slight green colour, and to greenish-brown after $24 \mathrm{hr}$. for all strains of $H$. vaginalis; a greenish area with haemolysis was most frequently observed, however. No haemolysis was produced by $\boldsymbol{H}$. aegyptius.

Maintenance on various media. The maintenance of Haemophilus vaginalis strains and one strain of $\boldsymbol{H}$. aegyptius was studied. The results of these studies are summarized in Table 1 from which it is apparent that whole blood or perhaps serum is necessary for the maintenance of Dukes, Edmunds, King and U/L strains. 

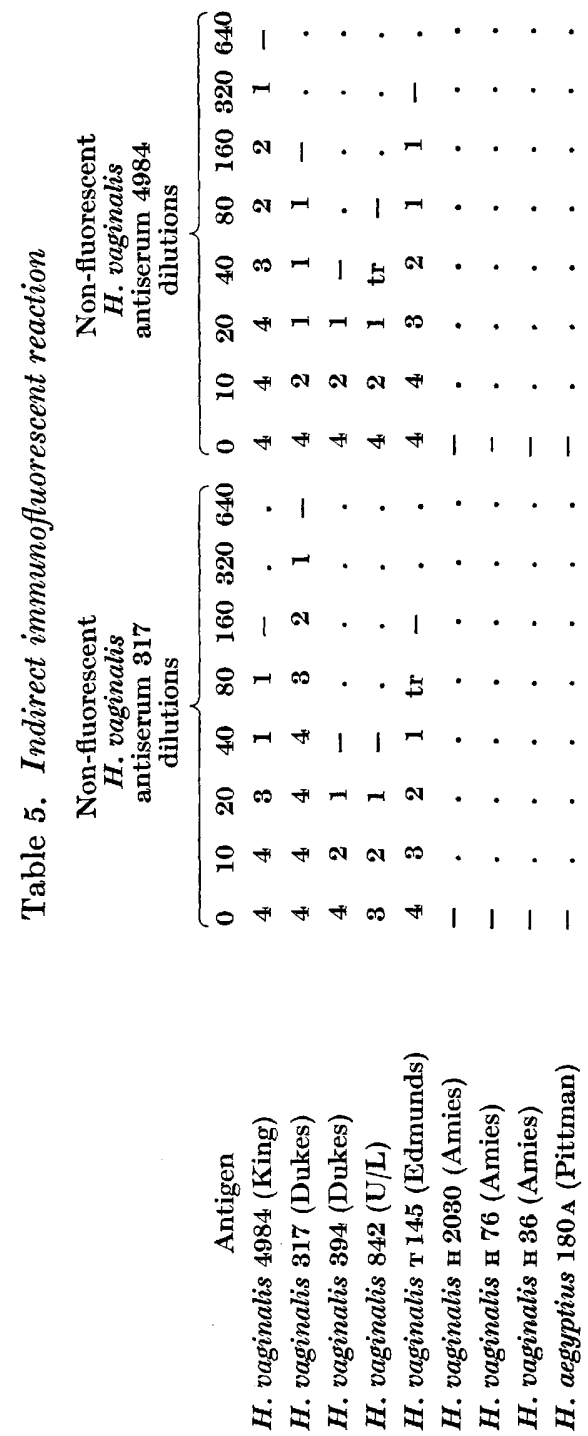





Agglutination tests. Results of tube agglutination reactions at $3 \mathrm{hr}$. and $18 \mathrm{hr}$. incubation are shown in Table 2 . The highest agglutination titres were obtained in homologous antigen and antiserum reactions. Significantly high titres were obtained, however, in some of the heterologous reactions with Haemophilus vaginalis. The titres of the Amies strains ( $\mathrm{H} 76$ and $\mathrm{H} 2030$ ) with the antisera of $H$. vaginalis 317, 4984 and 394 are not significant, although the titres for the $H$. aegyptius $180 \mathrm{~A}$ antiserum may be significant. Spontaneous agglutination of $H$. vaginalis $\mathrm{H} 36$ (Amies) was not avoided even when $0 \cdot 1 \%$ bovine serum albumin modified Hanks balanced salt solution was used; therefore, this strain was not included in the tube agglutination studies.

Immunofluorescent reactions. Specificity of the fluorescent Haemophilus vaginalis (317 and 4984) and $H$. aegyptius (180 A) antisera were determined by the inhibition reactions (see Table 3). Inhibition by non-fluorescent antiserum was greatest in the homologous antigen-globulin reaction. However, the non-fluorescent $H$. vaginalis antiserum reacted with heterologous $H$. vaginalis strains, resulting in inhibition of the fluorescent reaction. No significant inhibition was noted when non-fluorescent $H$. vaginalis 317 and 4984 antisera were mixed with $H$. aegyptius $180 \mathrm{~A}$ antigen.

The results of the cross-reaction studies by the direct method with species of Haemophilus are shown in Table 4. H. vaginalis fluorescent globulins 317 and 4984 reacted with homologous and heterologous $H$. vaginalis strains Dukes, Edmunds, King and $U / L$, but not with the Amies strains (н36, н 76, н 2030). These Amies strains did show a reaction with $H$. aegyptius fluorescent globulin $180 \mathrm{~A}$, as did $H$. influenzae strains and the $H$. aegyptius strains. Strains of $\boldsymbol{H}$. vaginalis (Dukes, King and $\mathrm{U} / \mathrm{L}$ ) other than those listed in Table 4 were also studied; all of these showed a fluorescent reaction of varying intensity with $H$. vaginalis fluorescent globulins 317 and 4984. No fluorescent reaction was observed with these strains when $H$. aegyptius fluorescent globulin $180 \mathrm{~A}$ was used. Indirect immunofluorescent reactions were made to further substantiate the specificity of direct and inhibition tests. The results are in Table $\mathbf{5}$.

\section{DISCUSSION}

The morphological and colonial characteristics of the Edmunds, King and U/L strains of Haemophilus vaginalis grown on Casman rabbit blood agar were consistent with the report of Dukes \& Gardner (1961). The characteristics of the Amies strains, however, differed on this medium, where they formed larger and umbonate colonies. Gram variability was noted in only two strains (Edmunds and King), and was not a consistent phenomenon in these two strains. In agreement with Dukes \& Gardner (1961), bipolar staining was observed frequently in Dukes, Edmunds, King and U/L strains of $H$. vaginalis, but was observed in only one Amies strain (H 2030).

All investigators with the exception of Amies \& Jones (1957) have noted that whole blood from one or another animal was a cultural requirement for strains of Haemophilus vaginalis. In the present work it was noted that Amies strains could be easily maintained on peptic digest agar and chocolate-yeastolate agar, both of which contain factors derived from whole blood. However, the Dukes, Edmunds, King and U/L strains could not be maintained on these media, thus indicating that 
some factor in whole blood or serum was needed by the latter strains, and was not provided by the whole blood derivatives.

Lapage (1961) used Casman agar in addition to other media in comparing his isolates with strains of Haemophilus vaginalis from Drs C. R. Amies and C. D. Dukes. Lapage established two cultural groups: (1) H. influenzae-like, which included the Amies strains of $H$. vaginalis; (2) $H$. vaginalis-like, which included a Dukes strain of this organism. On Casman rabbit blood agar a similar grouping was found in the present work, the Amies strains being similar to $H$. aegyptius in maintenance and morphology, while the Edmunds, King and U/L strains were identical with the strains of $\boldsymbol{H}$. vaginalis obtained from C. D. Dukes.

In the present work, the majority of strains produced a haemolysis as described by Dukes \& Gardner (1961); however, an occasional strain produced no haemolysis or produced an inner zone of $\beta$-haemolysis, in agreement with Edmunds (1960a). Because of these varied results, we believe that haemolysis is of little value in the identification of Haemophilus vaginalis colonies on Casman rabbit blood agar.

Colonies of Lactobacillus, Streptococcus and diphtheroids may be easily confused with Haemophilus vaginalis colonies macroscopically; it is therefore important to examine the minute colonies microscopically for granularity and irregular shape.

Haemophilus vaginalis is an extremely fastidious organism, and maintenance of stock cultures over long periods of time was difficult. Best results were obtained when the Dukes, Edmunds, King and U/L strains were transferred daily in Casman rabbit blood broth; other media suggested for the maintenance of the genus Haemophilus failed to support their growth. Casman rabbit blood broth and agar were used as the medium of choice for $H$. vaginalis. Amies strains differed from the above strains in that they could not be maintained in Casman rabbit blood broth; they were maintained successfully by transferring at 3- to 5-day intervals on chocolate-yeastolate agar or on Casman rabbit blood agar.

Dukes \& Gardner (1961) stated that the X and V factors are stimulatory accessory growth factors for Haemophilus vaginalis in the presence of partially digested protein, rabbit serum and certain amino acids. We found it impossible to supply the nutritional requirements of these strains by using a peptic digest prepared from whole rabbit blood or by using yeastolate with whole rabbit blood that had been denatured by heat. Thus, some factor or factors other than the $\mathrm{X}$ and $\mathrm{V}$ of whole blood are necessary for these strains. On the other hand, $\mathrm{X}$ and/or $\mathrm{V}$ factor requirements of Amies strains could be supplied by derivatives of whole rabbit blood such as a peptic digest of such blood or chocolate-yeastolate agar.

Agglutination reactions. The addition of $0 \cdot 1 \%$ bovine serum albumin to a modified balanced salt solution (e.g. Hanks) eliminated spontaneous agglutination in tube reactions with all strains of Haemophilus vaginalis except Amies strain $\mathrm{H36}$. No cross-reactions occurred with $\boldsymbol{H}$. influenzae, $\boldsymbol{H}$. aegyptius and Amies strains of $\boldsymbol{H}$. vaginalis and the three $H$. vaginalis antisera (317, 394 and 4984). Homologous and heterologous strains of $\boldsymbol{H}$. vaginalis (Edmunds, Dukes, King and $\mathrm{U} / \mathrm{L}$ ) were agglutinated by the three $H$. vaginalis antisera. In this investigation as in the investigation of Dukes \& Gardner (1961) the homologous strains produced high titres, whereas the heterologous strains produced lower titres.

Amies \& Jones (1957) did not observe cross-reactions with antisera for their strains of Haemophilus vaginalis and strains of H. influenzae and Bordetella pertussis 
antigens by the slide-agglutination test; however, they did not include $\boldsymbol{H}$. aegyptius in their investigation. $\boldsymbol{H}$. aegyptius antiserum agglutinated Amies strains of $\boldsymbol{H}$. vaginalis at titres of $1 / 80$ and did not agglutinate Dukes, Edmunds, King and U/L strains of $H$. vaginalis or $H$. influenzae. Pittman \& Davis (1950) stated that crossreactions with heterologous strains of $H$. aegyptius and $H$. aegyptius antisera at titres of $1 / 40$ were significant. In view of the cross-reactions observed with Amies strains of $\boldsymbol{H}$. vaginalis and $\boldsymbol{H}$. aegyptius antiserum in the present work, it appears that these strains are more closely related to $H$. aegyptius than to $H$. vaginalis.

Immunofluorescent reactions correlated with the agglutination reactions. Crossreactions did not occur in reactions with Haemophilus vaginalis antiserum (317 and 4984) and antigens (whole bacteria) of $H$. influenzae, H. aegyptius, and Amies strains of $\boldsymbol{H}$. vaginalis. Reactions did occur, however, with $\boldsymbol{H}$. vaginalis antiserum (317 and 4984) and any of the Dukes, Edmunds, King and U/L strains of $\boldsymbol{H}$. vaginalis. On the basis of these observations, it appears there are no common antigens shared by $H$. vaginalis, $H$. influenzae and $H$. aegyptius on the one hand and Amies strains of $\boldsymbol{H}$. vaginalis on the other. Cross-reactions did occur with $\boldsymbol{H}$. aegyptius fluorescent globulin and Amies strains of $H$. vaginalis and $H$. influenzae by the direct immunofluorescent method, thus indicating common antigens present in the three species. Further evidence of Amies strains of $H$. vaginalis sharing common antigens with $H$. aegyptius was provided by the indirect immunofluorescent method.

The inhibition and indirect immunofluorescent titres differed from the tubeagglutination titres with the same serum by a twofold or greater dilution factor. Such variation has been noted previously by Goldman (1957) during his investigation of Toxoplasma, and by Biegeleisen, Bradshaw \& Moody (1962) in their investigations of Brucella anti-bodies in human serum. Although such titres varied, the immunofluorescent reactions are considered to be highly specific.

The specificity of the fluorescent Haemophilus vaginalis (317 and 4984) globulin was determined by inhibition and direct immunofluorescent methods. Crossreactions which occurred with other species of Haemophilus and undiluted fluorescent globulin were avoided by diluting the fluorescent antisera 1/12 in the direct immunofluorescent method.

This investigation was supported by a grant, AI-1530, from the National Institute of Allergy and Infectious Diseases, NIH, U.S. Public Health Service.

Clinical co-operation from L. A. Gray and L. O. Giesel, Jr. (Louisville, Kentucky) and from H. L. Gardner (Houston, Texas), is gratefully acknowledged. The authors are also grateful to Dr C. D. Dukes, Baylor University, College of Medicine, Houston, Texas, for making available certain cultures and laboratory facilities for work done in Houston. 


\section{REFERENCES}

Amies, C. R. \& Jones, S. A. (1957). A description of Haemophilus vaginalis and its Lforms. Canad. J. Microbiol. 3, 579.

Biegeleisen, J. Z., Jr., Bradshaw, B. R. \& Moody, M. D. (1962). Demonstration of Brucella antibodies in human serum. A comparison of the fluorescent antibody and agglutination techniques. J. Immunol. 88, 109.

Brewer, J. I., Halpern, B. \& Thomas, G. (1957). Haemophilus vaginalis vaginitis. Amer. J. Obstet. Gynec. 74, 834.

Casman, E. P. (1947). A noninfusion blood agar base for Neisseria, Pneumococci, and Streptococci. Amer. J. clin. Path. 17, 281.

Cherry, W. B., Goldman, M., Carski, T. \& Moody, M. D. (1960). Fluorescent antibody techniques in the diagnosis of communicable diseases. Publ. Hth Serv. Publ. Wash. no. 792

Dukes, C. D. \& Gardner, H. L. (1961). Identification of Haemophilus vaginalis. J. Bact. 81, 277.

Edmunds, P. N. (1960a). Haemophilus vaginalis: morphology, cultural character and viability. J. Path. Bact. 79, 273.

Eomunds, P. N. (1960b). The growth requirements of Haemophilus vaginalis. J. Path. Bact. 80, 325.

Evans, E. E. (1957). Serological Methods. Manual of Microbiological Methods, 5th ed. New York: McGraw-Hill Book Co.

Fildes, P. (1920). A new medium for the growth of B. influenzae. Brit. J. exp Path. $1,129$.

Gardner, H. L. \& DUkes, C. D. (1954). New etiologic agent in non-specific vaginitis. Science, 120, 853.

Gardner, H. L. \& Dukes, C. D. (1955). Haemophilus vaginalis vaginitis: A newly defined specific infection, previously classified as non-specific vaginitis. Amer. $J$. Obstet. Gynec. 69, 962.

Gardner, H. L., Dampeer, T. K. \& Dukes, C. D. (1957). The prevalence of vaginitis. A study in incidence. Amer. J. Obstet. Gynec. 73, 1080.

Goldman, M. (1957). Staining Toxoplasma gondii with fluorescein-labelled antibody. II. A new serologic test for antibodies to Toxoplasma based upon inhibition of specific staining. J. exp. Med. 105, 557.

LAPAGE, S. P. (1961). Haemophilus vaginalis and its role in vaginitis. Acta path. microbiol. scand. $52(1), 34$.

LEOPOLD, S. (1953). Heretofore undescribed organism isolated from the genitourinary system. U.S. Forces med. J. 4, 263.

Lutz, A. \& Burger, P. (1957). In Les Infections a Trichomonas. Premier Symposium Européen. Paris: Masson et Cie.

Pittman, M. \& Davis, D. J. (1950). Identification of the Koch-Weeks bacillus (Haemophilus aegyptius). J. Bact. 59, 413. 